\title{
Appearance of quark-hadron duality in the Rein-Sehgal model
}

\author{
Krzysztof M. Graczyk, Cezary Juszczak, Jan T. Sobczyk a* \\ ${ }^{a}$ Institute of Theoretical Physics \\ University of Wrocław \\ pl. M. Borna 9, 50-204, Wrocław, Poland
}

Quark-hadron duality in neutrino-nucleon reactions is investigated under the assumption that cross sections in the resonance region are given by the Rein-Sehgal model. The quantitative analysis of the duality is done by means of appropriate integrals of the structure functions in the Nachtmann variable. We conclude that with the definition of the resonance region $W \in\left(M+m_{\pi}, 2 \mathrm{GeV}\right)$ the duality holds for neutrino-proton reaction $F_{2}$ structure function for $Q^{2} \in(0.5,3) \mathrm{GeV}^{2}$ and it is absent for neutrino-neutron reaction.

\section{INTRODUCTION}

The discovery of quark-hadron duality in electron-nucleon interactions [1] rises a natural question if the same phenomenon can be seen also in neutrino-nucleon scattering. Unfortunately the available data is not yet precise enough to discuss the problem on the experimental level. At present the only possibility is to analyze the existing theoretical models of resonance production. This approach was adopted by Sato and Lee [2] who discovered that for their model of $\Delta$ production the quark-hadron duality is seen: the resonance peaks of structure functions calculated at $Q^{2}=0.4,1,2,4 \mathrm{GeV}^{2}$ slide along the DIS structure functions (with CTEQ6 PDFs) calculated at $10 \mathrm{GeV}^{2}$, both as functions of the Nachtmann variable.

In this contribution we wish to report an investigation of the Rein-Sehgal (RS) model 3 of resonance production. The RS model is used by almost all Monte Carlo generators of neutrino interactions and understanding its properties is of practical value. The model describes resonance production in the region of hadronic invariant mass $W$ below $2 \mathrm{GeV}$ by summing contributions from 18 resonances. It contains also a non-resonant background fine tuned in order to get a good agreement with the existing single pion production (SPP) data.

\footnotetext{
${ }^{*}$ The authors were supported by KBN grant 105/E-
} 344/SPB/ICARUS/P-03/DZ211/2003-2005
We calculate the structure functions $F_{1}, F_{2}, F_{3}$ as they are defined by the RS model 4]. They turn out to be linear combinations of cross sections for three polarization states of the intermediate $W$ boson. The aim of the RS model is to describe only SPP channels and the elasticity coefficients are used in order to select exclusive SPP channels from the overall resonance production cross section. The non-resonant background is adjusted only for SPP channels. The analysis of duality requires the knowledge of the inclusive cross section in the resonance region. In the spirit of the RS model one should add contributions from inelastic channels taking care of complicated interference patterns. In order to make the analysis simpler we introduce $1 \pi$ functions which are defined as probabilities that in the given region of the kinematically allowed space the final state is that of SPP. We calculate them numerically for each SPP channel separately using the LUND fragmentation and hadronization routines. All the details of our approach can be found in 4 .

\section{FORMALISM AND RESULTS}

The $1 \pi$ functions are defined (for each exclusive channel separately) as follows :

$$
f_{1 \pi}\left(W, Q^{2}\right) \equiv \frac{\frac{d^{2} \sigma^{S P P}}{d W d Q^{2}}}{\frac{d^{2} \sigma^{D I S}}{d W d Q^{2}}}
$$




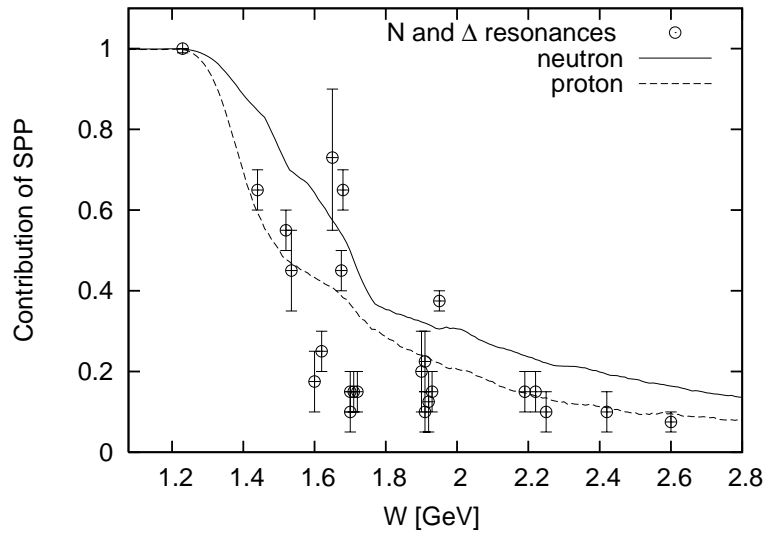

Figure 1. A comparison of $1 \pi$ functions defined in Eq. 1 with resonance elasticity factors taken from PDG [5]. In the case of $\nu$-neutron scattering we show the sum of two functions corresponding to $\nu_{\mu} n \rightarrow$ $\mu^{-} p \pi^{0}$ and $\nu_{\mu} n \rightarrow \mu^{-} n \pi^{+}$reactions

It turns out that $f_{1 \pi}\left(W, Q^{2}\right)$ do not depend on $Q^{2}$. Their plots for $\mathrm{CC} \nu N$ channels are presented in Fig. 1 In the case of neutron we show the sum of $1 \pi$ functions corresponding to two exclusive SPP channels. On the same plot we show also the available data on elasticity of $N$ and $\Delta$ resonances. We see that $1 \pi$ functions provide satisfactory average description.

We re-scale RS model structure functions and obtain approximations of structure functions in the RES region:

$F_{i}^{R E S}\left(x, Q^{2}\right)=\frac{F_{i}^{R S}\left(x, Q^{2}\right)}{f_{1 \pi}\left(x, Q^{2}\right)}, \quad i=1,2,3$.

In our quantitative analysis we define the following function:

$\mathcal{R}_{2}\left(Q^{2} ; Q_{D I S}^{2}\right)=\frac{\int_{\xi_{\min }}^{\xi_{\max }} d x F_{2}\left(\xi, Q^{2}\right)}{\int_{\xi_{\min }}^{\xi_{\max }} d \xi F_{2}^{D I S}\left(\xi, Q_{D I S}^{2}\right)}$.

$\xi$ is the Nachtmann variable. $\xi_{\max , \min }$ are defined by the conditions $W\left(\xi_{\max }, Q^{2}\right)=M+m_{\pi}$ $W\left(\xi_{\min }, Q^{2}\right)=W_{\max }$. We investigate three

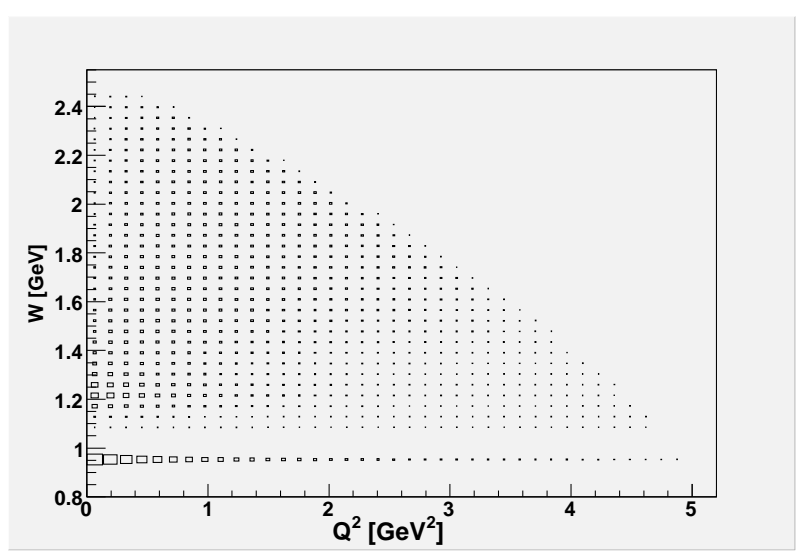

Figure 2. Double differential cross section $\frac{d^{2} \sigma}{d W d Q^{2}}$ for neutrino energy $3 \mathrm{GeV}$.

choices for $W_{\max }$. The perfect duality would mean that $\mathcal{R}_{2}\left(Q^{2} ; Q_{D I S}^{2}\right) \simeq 1$.

We select the physically relevant region in the $Q^{2}$ variable as $Q^{2} \leq 3 \mathrm{GeV}^{2}$ by looking at double differential cross section $\frac{d^{2} \sigma}{d W d Q^{2}}$ for neutrino energy in a few GeV region, see Fig. 2 [6]. Having in mind MC generators of events the quark-hadron duality should ensure the smooth passage of the cross section for RES and DIS contributions in the relevant kinematical transition region.

In Fig. [3 we show $F_{2}$ structure functions for CC reactions defined by the RS model for three values of $Q^{2}: 0.4,1,2 \mathrm{GeV}^{2}$ and also DIS $F_{2}$ structure functions calculated at $Q^{2}=10 \mathrm{GeV}^{2}$. The targets are from the top to the bottom: proton, neutron and their average isoscalar one. The plots for the DIS part are done with GRV94 PDFs 7 . RS structure functions are yet not re-scaled by $1 \pi$ functions. Similar plots for $F_{2}$ at $Q^{2}=0.5,1$ $\mathrm{GeV}^{2}$ were shown before in 8 . We see that in the case of proton and isoscalar target typical manifestation of local quark-hadron duality: the $\Delta$ peak slides along the DIS curve. The duality does not seem to apply neither to other resonances nor to neutron target even in the case of the prominent $\Delta$ resonance.

In Fig. 4 we show the role played by $1 \pi$ functions. On the same plot both Rein-Sehgal and $1 \pi$ 

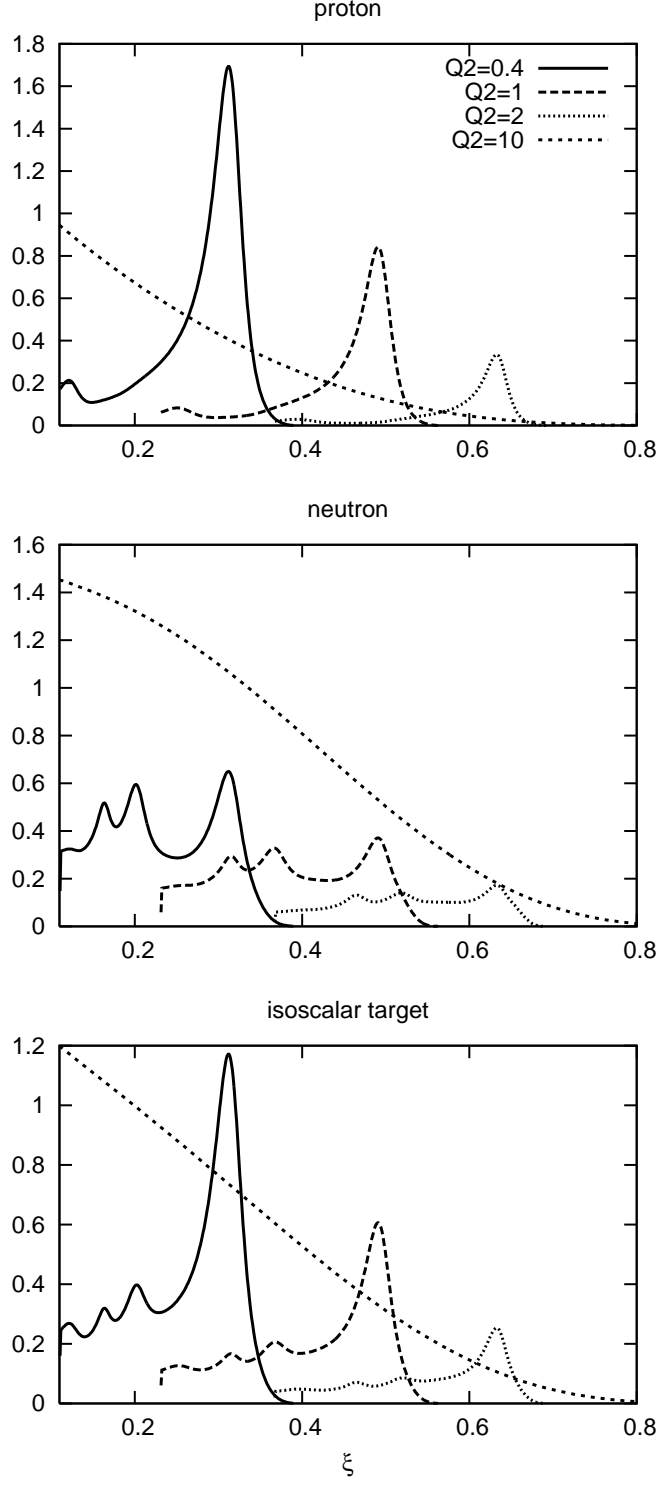

Figure 3. Manifestation of quark-hadron duality for $F_{2}$ structure function for $\mathrm{CC}$ neutrino reactions on proton, neutron and isoscalar target. $F_{2}$ for RS model is calculated at $Q^{2}=0.4,1,2 \mathrm{GeV}^{2}$ and for DIS at $10 \mathrm{GeV}^{2}$.

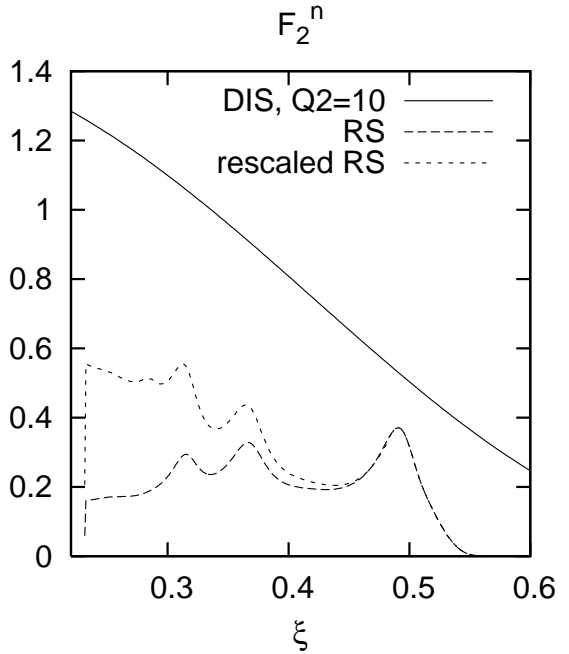

Figure 4. The comparison of $F_{2}$ structure functions for the RS model, re-scaled RS model and the DIS. The structure functions are calculated for CC $\nu$-neutron scattering at $Q^{2}=1 \mathrm{GeV}^{2}$.

function re-scaled RES $F_{2}$ structure functions for $\mathrm{CC}$ reaction on neutron are presented. We see that the modifications apply mostly to the region of $W$ close to $2 \mathrm{GeV}$.

Fig. [5]presents the functions defined in Eq. 3 for three different integration regions i.e. for three definitions of the resonance region: $W \in(M+$ $\left.m_{\pi}, W_{\max }\right)$ where $W_{\max }=1.6,1.8,2 \mathrm{GeV}$. We see that the choice $W_{\max }=2 \mathrm{GeV}$ makes the functions slowly varying in the wide region of $Q^{2}$. The behavior for small values of $Q^{2} \leq 0.5 \mathrm{GeV}^{2}$ is very different in agreement with the predictions made in [9]. For the proton target the best choice is $W_{\max }=2 \mathrm{GeV}$ while for other two targets it is preferable to choice the resonance region as more confined at the price of significant variations with $Q^{2}$. The choice $W_{\max }=2 \mathrm{GeV}$ is a natural one for the RS model. But it is suggested in [10] that the RS model underestimates the cross section for $W \geq 1.7 \mathrm{GeV}$. We conclude that with the choice $W_{\max }=2 \mathrm{GeV}$ the duality holds very well for the proton and badly for other targets. It seems to be difficult to have duality for all the targets 
simultaneously.

\section{Acknowledgments}

We thank Olga Lalakulich for a discussion on resonance elasticities and Jarosław Nowak for preparing for us the Figs. 1] and 2

\section{REFERENCES}

1. For a general review see: W. Melnitchouk, R. Ent, and C. E. Keppel, Phys. Rep. 406 (2005) 127.

2. K. Matsui, T. Sato, and T.-S. H. Lee, Phys. Rev. C 72 (2005) 25204.

3. D. Rein, L. M. Sehgal, Ann. Phys. 133, (1981) 79 .

4. K. M. Graczyk, C. Juszczak, and J. T. Sobczyk, Quark-hadron duality in the ReinSehgal model, hep-ph/0512015

5. The Review of Particle Physics, http://pdg.lbl.gov/.

6. The model is described in: C. Juszczak, J. A. Nowak, and J. T. Sobczyk, Simulations from a new neutrino event generator, these proceedings.

7. M. Glück, E. Reya, and A. Vogt, Z. Phys. C 67 (1995) 433.

8. P. Lipari, Calculation of the neutrino cross section. Open problems, lines of research a talk given at Third International Workshop on Neutrino-Nucleus Interactions in the FewGeV Region, Assergi, March 17-21, 2004.

9. F. E. Close, N. Isgur, Phys. Lett. B 509, 81 (2001).

10. A. Bodek, NuInt02 Conference Summary: Modelling Quasi-elastic, Resonance and Inelastic Neutrino and Electron Scattering on Nucleons and Nuclei, a talk at Second International Workshop on Neutrino-Nucleus Interactions in the Few-GeV Region, Irvine, Dec 12-15, 2002.
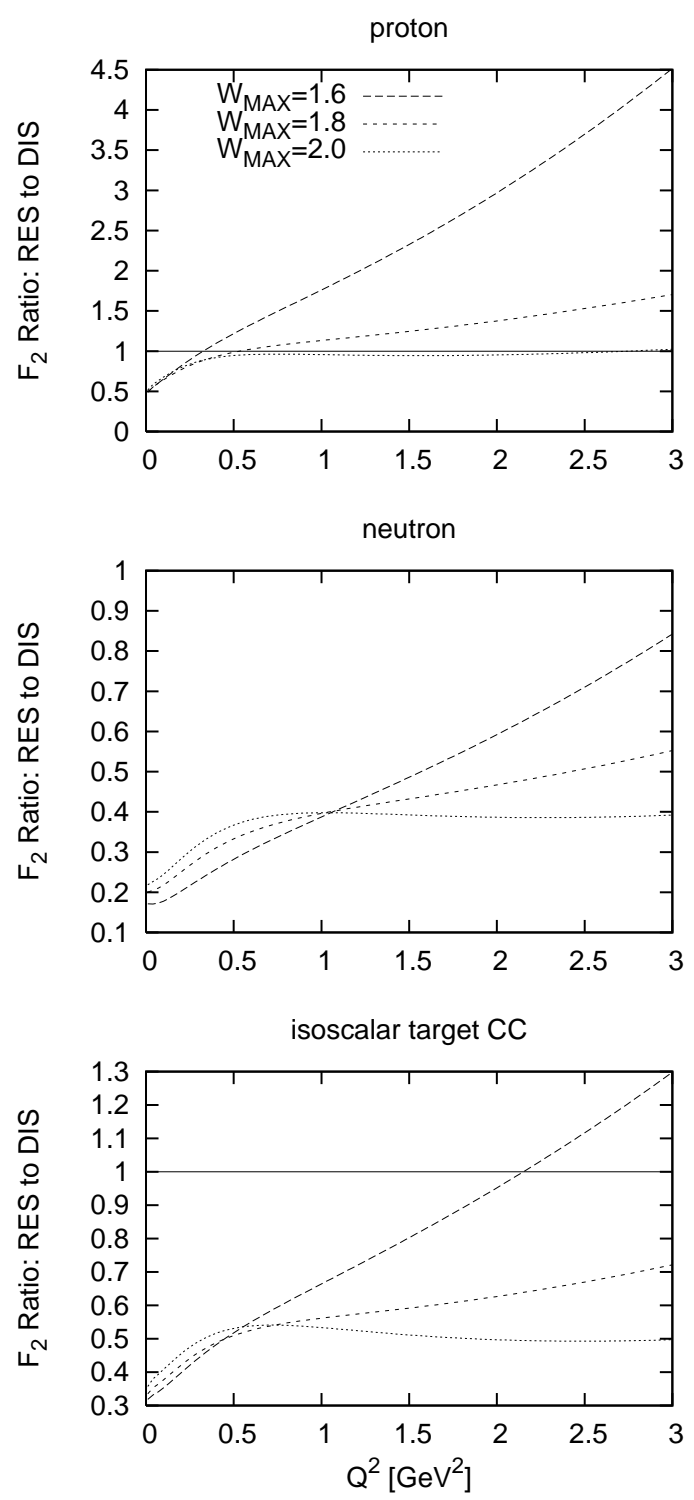

Figure 5. Function $\mathcal{R}_{2}\left(Q^{2} ; Q_{D I S}^{2}=10 \mathrm{GeV}^{2}\right)$ (see Eq. 3) for CC reaction on proton, neutron and isoscalar targets with $W_{\max }=1.6,1.8,2.0 \mathrm{GeV}$. 